\title{
Cardiac damage related to shotgun injury: Case report
}

\author{
Habib Cakir*, Hasan Uncu, Cagatay Tuncel, Ahmet Cakallioglu, Ozsoyler Ibrahim \\ Department of Cardiovascular Surgery, Adana Numune Education and Training Hospital, Adana, Turkey \\ Email: *habibcakir35@hotmail.com
}

Received 8 May 2012; revised 17 June 2012; accepted 28 June 2012

\begin{abstract}
In this report, we present the case of a patient who presented with a pellet in his myocardium and was managed with cardiac tamponade due to shotgun injury. A 49-year-old man presented to the emergency department due to a close-range shotgun pellet injury. The patient was operatedemergently because arterial pressure was progressively decreasing and cardiac tamponade was detected echocardiographically. Three pellet entrance hole were detected on left ventricule posterior wall in surgical exploration after the cardiac tamponade relieved. No further intervention was considered because bleeding discontinued. We conclude that in shotgun pellet cardiac injuries, if the pellets are located in the myocardium, the operational decision and surgical procedure to be undertaken depends on the clinical situation of the patient and the echocardiographic signs.
\end{abstract}

Keywords: Shotgun; Heart Injuries; Cardiactamponade

\section{INTRODUCTION}

Cardiac injuries can result from penetrating or blunt traumas. Since they need urgent diagnosis and treatment, these injuries are of prime importance among thoracic traumas. Common penetrating traumas may result from sharp items, shotguns, or iatrogenic reasons. Though no incidence rate is proven for this type, almost half of these patients are known to die at the accident scene. Shotgun injuries are different from the others in that the pellets inflict a great amount of energy onto the tissues and thus the majority of the injury occurs on the tissues. In this report, we present the case of a patient who presented with a pellet in his myocardium and was managed with cardiac tamponade due to shotgun injury.

\section{CASE REPORT}

A 49-year-old man presented to the emergency department due to a close-range shotgun pellet injury. Upon the detection of hemathorax, a tube thoracostomy was app-

${ }^{*}$ Corresponding author. lied to the left hemathorax. Due to intraabdominal bleeding, the patient was urgently managed with primary repair by the department of general surgery. The patient was transferred to a hospital with thoracic surgery clinic due to hemathorax. A thoraco-abdominal tomography both visualized the presence of pericardial fluid and detected the great number of pellets both in the lungs and in the myocardium. Depending on these signs, a cardiovascular consultation was obtained. In physical examination, tension arterial was $90 / 50 \mathrm{~mm}$ HG and the pulse rate was 110 beats/min. Also, there were many pellet holes in the left hemathorax posterior wall. No foreign body was detected in the ventricules during transthoracic echocardiography. The patient was urgently operated on when tamponade was detected and tension arterial decreased. Following the median sternotomy, the pericardium was opened and tamponade became visible. Hemorrhagic fluid was drained. During the explorations, three pellet holes with no hemorrhage were detected on the left ventricule posterior wall. The holes were managed with biological tissue adhesives. Both hemathoraxes were opened thereafter. In both lungs there existed countless pellet holes with no active hemorrhage. Postoperatively no additional pathology was observed in lung graphy, transthoracic echocardiography, and thoraco-abdominal tomography (Figures 1-3). The patient was uneventfully discharged on the eleventh postoperative day. Seven days later, the presence of pellets in the myocardium was viewed by a transthoracic echocardiography.

\section{DISCUSSION}

Penetrating cardiac traumas are quite rare. The incidence rate for these traumas cannot be assessed since the actual number of patients that die before hospital admission is unknown. According to a study, sharp items are the most common causes for penetrating cardiac traumas [1]. On the other hand, shotgun injuries convey the highest mortality rate [1]. Our patient had a shotgun injury but he did not develop mortality.

Since they have a high risk of mortality, patients with cardiac penetrating injuries should be urgently diagnosed and managed. However, delayed diagnosis may occur 


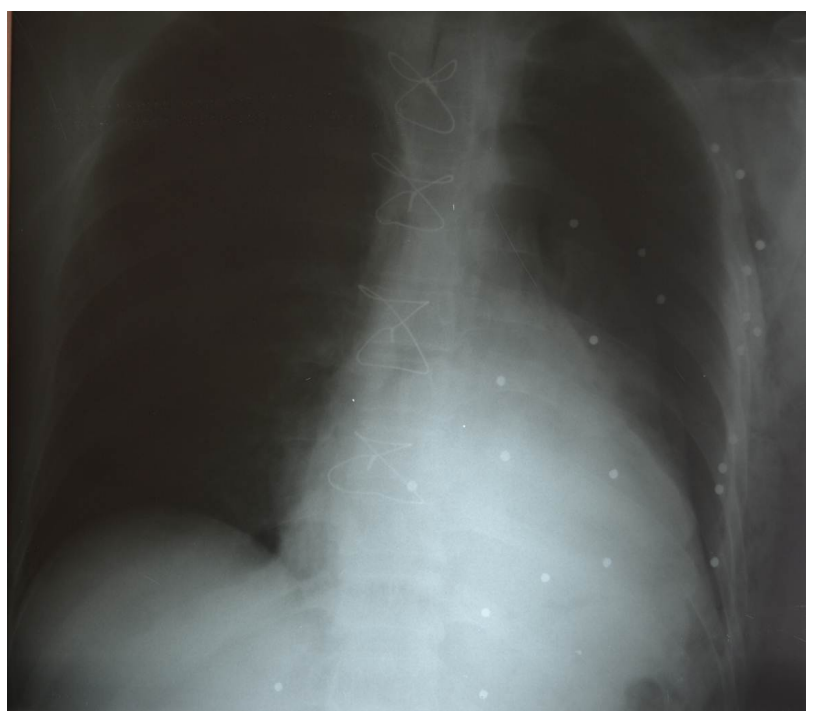

Figure 1. The postoperative PA chest roentgenography (three pellet on the left ventricule posterior wall were marked by arrows).

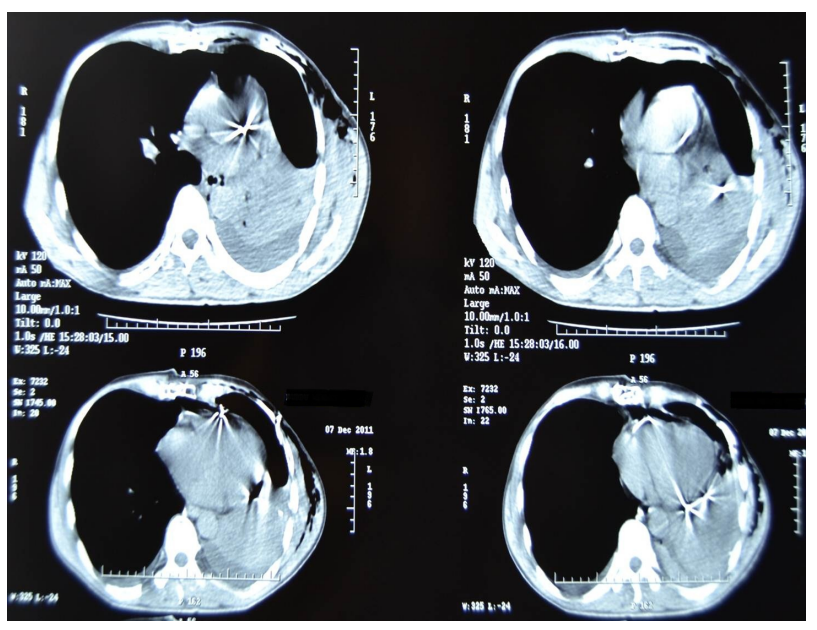

Figure 2. The postoperative thoracic computerised tomographysections (two pellet on the left ventricule posterior wall were seen in right inferior imaging).

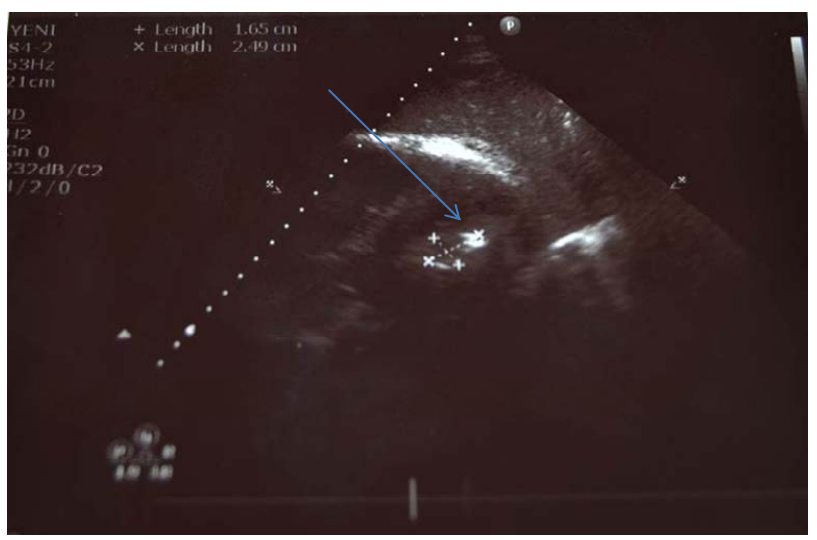

Figure 3. The echocardiographic image of the pellet in the myocardial tissue was marked by arrow. since these cases are quite rare. Most of these patients are hemodynamically instable, and thus there is no enough time for further diagnostic tests. Although he had cardiac injury, our patient was only diagnosed through further diagnostic tests following colon surgery. Indeed, the cardiovascular consultation was delayed as well. The period between the time of shooting and the cardiovascular consultation was about 8 hours, which we consider to be too long. Therefore, particularly in penetrating shotgun thoracic injuries, the cardiac injury should initially be managed. Also, we consider that well-trained surgeons at the emergency departments can reduce the risk of mortality in such cases. Remarkably, there are some studies reporting that the tamponade that results from penetrating cardiac injuries has a positive effect on mortality [2]. Our patient was present with tamponade as well, but he did not develop mortality. Although he had a cardiac injury, the patient was hemodynamically stable throughout the 8 hours, which we attribute to the fact that 3 pellets had stayed at the myocardium and did not penetrate into heart chambers.

Shotgun cardiac injuries differ from other cardiac traumas in terms of clinical signs and treatment strategies. In shotgun cardiac injuries, since the pellet(s) remain in the parenchyma, the procedure to be undertaken depends on the clinical situation of the patient and the amount of pericardial fluid. In the literature, numerous procedures are reported for such cases, such as follow-up, subxiphoid pericardial window, thoracotomy, and sternotomy. Follow-up can be preferred for hemodynamically stable patients who have no pericardial fluid [3]. Sternotomy was performed in our patients because he presented with tamponade in transthoracic echocardiography, had reduced tension arterial, and was injured at the posterior. The explorations revealed three pellet holes in the left ventricule posterior wall. No further surgery was performed for our patient since no foreign body was detected in the preoperative echocardiography, no hemorrhage occurred in the pellet holes, and the pellets had penetrated into the myocardium. There have been a number of studies preferring peripheral emboli for shotgun pellet cardiac injuries [4]. In our patient, no embolic signs were detected both pre- and post-operatively. Preoperatively some patients may feel extremely nervous knowing that they are bearing a foreign body in their cardiac tissues. In this case, while removing the pellets, a further procedure may become vital [5]. No such cases occurred with our patient as he was informed about the process in advance.

In the cases of thoracic shotgun injury, cardiac injury should be initially managed even if the patients are hemodynamically stable. Any other procedure might prove inessential and thus may increase the risk of mortality. We conclude that in shotgun pellet cardiac injuries, if the pellets are located in the myocardium, the operational 
decision and surgical procedure to be undertaken depend on the clinical situation of the patient and the echocardiographic signs.

\section{REFERENCES}

[1] Manduz, S., Katrancioglu, N., Bingol, H., Atli, H. and Dogan, K. (2008) Penetrating cardiac injuries. Türk Göğ̈̈s Kalp Damar, 16, 228-231.

[2] Moreno, C., Moore, E.E., Majure, J.A. and Hopeman, A.R. (1986) Pericardial tamponade: A critical determinant for survival following penetrating cardiac wounds. Journal of Trauma, 26, 821-825. doi:10.1097/00005373-198609000-00007

[3] Alejandro, V.K., Acosta, A.J. and Rodriguez, A.P. (2003) Air gun pellet cardiac injuries: Case report and rewiew of the literature. Journal of Trauma, 54, 1242-1244.

[4] Schowengerdt, C.G., Vasko, J.S., Craenen, J.M. and Teske, D.W. (1985) Air gun pellet injury of the heart with popliteal embolus. Annals of Thoracic Surgery, 40, 393395. doi:10.1016/S0003-4975(10)60076-8

[5] Ebell, B.J., Graft, B., Sievers, H.H. and Duebener, F.L. (2008) An air gun pellet retained in the heart: A case report. Heart Surgery Forum, 11, 127-128. doi:10.1532/HSF98.20071208 